\title{
The winding road to quantum gravity
}

\author{
Abhay Ashtekar ${ }^{1,2,3}$ \\ ${ }^{1}$ Institute for Gravitational Physics and Geometry, Physics Department, 104 Davey, Penn State, University Park, PA 16802, USA \\ ${ }^{2}$ Max Planck Institut für Gravitationsphysik, Albert Einstein Institut, 14476 Golm, Germany \\ ${ }^{3}$ Indian Academy of Sciences, Sir C. V. Raman Road, Bangalore 560 080, India
}

\begin{abstract}
A brief history of quantum gravity is sketched. While familiarity with basic ideas and notions of contemporary physics is assumed, technicalities are kept to a minimum and use of equations is avoided. Rather, the emphasis is on providing a coherent picture of the evolution of ideas and the current status of the subject.
\end{abstract}

Keywords: General relativity, quantum gravity, string theory.

\section{The beginning}

GENERAL relativity and quantum theory are among the greatest intellectual achievements of the 20th century. Each of them has profoundly altered the conceptual fabric that underlies our understanding of the physical world. Furthermore, each has been successful in describing the physical phenomena in its own domain to an astonishing degree of accuracy. And yet, they offer us strikingly different pictures of physical reality. Indeed, at first one is surprised that physics could keep progressing blissfully in the face of so deep a conflict. The reason is that phenomena for which both theories are essential occur at the Planck scale and the values of fundamental constants in our universe conspire to make the Planck length $\ell_{\mathrm{Pl}}=$ $\sqrt{G \hbar / c^{3}} \sim 10^{-33} \mathrm{~cm}$ truly minute and Planck energy $E_{\mathrm{Pl}}=\sqrt{\hbar c / G} \sim 10^{19} \mathrm{Gev}$ absolutely enormous compared to laboratory scales. Thanks to this coincidence, we can happily maintain a schizophrenic attitude and use the precise, geometric picture of reality offered by general relativity while dealing with cosmological and astrophysical phenomena, and the quantum-mechanical world of chance and intrinsic uncertainties while dealing with atomic and subatomic particles. Clearly, this strategy is quite appropriate as a practical stand. But it is highly unsatisfactory from a conceptual viewpoint. Everything in our past experience in physics tells us that the two pictures we currently use must be approximations, special cases that arise as appropriate limits of a grander theory. That theory must therefore represent a synthesis of general relativity and quantum mechanics. This would be the quantum theory of gravity. The burden on this theory is huge: Not only should it correctly describe all the known gravitational

e-mail: ashtekar@gravity.psu.edu processes, but it should also adequately handle the Planck regime. This is the theory that we invoke when faced with phenomena, such as the big bang and the final state of black holes, where the Planck scale is reached and worlds of general relativity and quantum mechanics unavoidably meet.

It may come as a surprise that the necessity of a quantum theory of gravity was pointed out by Einstein already in 1916 - barely a year after the discovery of general relativity. In a paper in the Preussische Akademie Sitzungsberichte, he wrote:

'Nevertheless, due to the inneratomic movement of electrons, atoms would have to radiate not only electromagnetic but also gravitational energy, if only in tiny amounts. As this is hardly true in Nature, it appears that quantum theory would have to modify not only Maxwellian electrodynamics but also the new theory of gravitation'.

Papers on the subject began to appear in the thirties most notably by Bronstein, Rosenfeld and Pauli. However, detailed work began only in the sixties. The general developments since then loosely represent four stages, each spanning roughly a decade.

First, there was the beginning: exploration. The goal was to do unto gravity as one would do unto any other physical field $^{1}$ (Since this article is addressed to non-experts, except in the discussion of very recent developments, I will generally refer to books and review articles which summarize the state of the art at various stages of development of quantum gravity. References to original papers can be found in these reviews.). The electromagnetic field had been successfully quantized using two approaches: $c a$ nonical and covariant. In the canonical approach, electric and magnetic fields obeying Heisenberg's uncertainty principle are at the forefront, and quantum states naturally arise as (gauge-invariant) functions $\psi(A)$ of the vector potential $A$ on a constant time 3 -surface of space-time. In the covariant approach on the other hand, one first isolates and then quantizes the two radiative modes of the Maxwell field in space-time, without carrying out a $(3+1)$ decomposition of space-time into space and time. The quantum states naturally arise as elements of the Fock space of photons. Attempts were made to extend these techniques to general relativity. In the electromagnetic 
case the two methods are completely equivalent. Only the emphasis changes in going from one to another. In the gravitational case, however, the difference is profound. This is not accidental. The reason is deeply rooted in one of the essential features of general relativity, namely the dual role of the space-time metric.

To appreciate this point, let us begin with field theories in Minkowski space-time, say Maxwell's theory to be specific. Here, the basic dynamical field is represented by a tensor field $F_{\mu \nu}$ on Minkowski space. The space-time geometry provides the kinematical arena on which the field propagates. The background, Minkowskian metric provides light cones and the notion of causality. We can foliate this space-time by a one-parameter family of constanttime three-planes, and analyse how the values of electric and magnetic fields on one of these surfaces determine those on any other surface. The isometries of the Minkowski metric let us construct physical quantities such as fluxes of energy, momentum, and angular momentum carried by electromagnetic waves. Geometry of Minkowski space, on the other hand, is fixed; it is completely insensitive to the properties of the electromagnetic field.

In general relativity, by contrast, there is no background geometry. The space-time metric itself is the fundamental dynamical variable. On the one hand it is analogous to the Minkowski metric in Maxwell's theory; it determines space-time geometry, provides light cones, defines causality, and dictates the propagation of all physical fields (including itself). On the other hand it is the analog of the Newtonian gravitational potential and therefore the basic dynamical entity of the theory, similar in this respect to the vector potential $A_{\mu}$ of the Maxwell theory. This dual role of the metric is in effect a precise statement of the equivalence principle that is at the heart of general relativity. It is this feature that is largely responsible for the powerful conceptual economy of general relativity, its elegance, its aesthetic beauty, its strangeness in proportion.

However, this feature also brings with it a host of problems. We see already in the classical theory several manifestations of these difficulties. It is because there is no background geometry, for example, that it is so difficult to analyse singularities of the theory and to define the energy and momentum carried by gravitational waves. Since there is no a priori space-time, to introduce notions as basic as causality, time, and evolution, one must first solve the dynamical equations and construct a space-time. As an extreme example, consider black holes, whose definition requires the knowledge of the causal structure of the entire space-time. To find if the given initial conditions lead to the formation of a black hole, one must first obtain their maximal evolution and, using the causal structure determined by that solution, ask if its future infinity has a past boundary. If it does, space-time contains a black hole and the boundary is its event horizon. Thus, because there is no longer a clean separation between the kinematical arena and dynamics, in the classical theory substan- tial care and effort is needed even in the formulation of basic physical questions.

In quantum theory the problems become significantly more serious. To see this, recall first that, because of the uncertainty principle, already in non-relativistic quantum mechanics particles do not have well-defined trajectories; time-evolution only produces a probability amplitude, $\psi(x, t)$, rather than a specific trajectory, $x(t)$. Similarly, in quantum gravity, even after evolving an initial state, one would not be left with a specific space-time. In the absence of a space-time geometry, how is one to introduce even habitual physical notions such as causality, time, scattering states, and black holes?

\section{Early developments}

The canonical and the covariant approaches have adopted dramatically different attitudes to face these problems. In the canonical approach, one notices that, in spite of the conceptual difficulties mentioned above, the Hamiltonian formulation of general relativity is well-defined and attempts to use it as a stepping stone to quantization. The fundamental canonical commutation relations are to lead us to the basic uncertainty principle. The motion generated by the Hamiltonian is to be thought of as time evolution. The fact that certain operators on the fixed ('spatial') threemanifold commute is supposed to capture the appropriate notion of causality. The emphasis is on preserving the geometrical character of general relativity, on retaining the compelling fusion of gravity and geometry that Einstein created. In the first stage of the program, completed in the early sixties, the Hamiltonian formulation of the classical theory was worked out in detail by Dirac, Bergmann, Arnowitt, Deser and Misner and others ${ }^{2-6}$. The basic canonical variable was the 3-metric on a spatial slice. The ten Einstein's equations naturally decompose into two sets: four constraints on the metric and its conjugate momentum (analogous to the equation Div $\vec{E}=0$ of electrodynamics) and six evolution equations. Thus, in the Hamiltonian formulation, general relativity could be interpreted as the dynamical theory of 3-geometries. Wheeler therefore baptized it geometrodynamics ${ }^{7,8}$.

In the second stage, this framework was used as a point of departure for quantum theory. The basic equations of the quantum theory were written down and several important questions were addressed $^{6,8}$. Wheeler also launched an ambitious program in which the internal quantum numbers of elementary particles were to arise from non-trivial, microscopic topological configurations and particle physics was to be recast as 'chemistry of geometry'. However, most of the work in quantum geometrodynamics continued to remain formal; indeed, even today the field theoretic difficulties associated with the presence of an infinite number of degrees of freedom remain unresolved. Furthermore, even at the formal level, it has been difficult to 
solve the quantum Einstein's equations. Therefore, after an initial burst of activity, the quantum geometrodynamics program became stagnant. Interesting results were obtained in the limited context of quantum cosmology where one freezes all but a finite number of degrees of freedom. However, even in this special case, the initial singularity could not be resolved without additional 'external' inputs into the theory. Sociologically, the program faced another limitation: concepts and techniques which had been so successful in quantum electrodynamics appeared to play no role here. In particular, in quantum geometrodynamics, it is hard to see how gravitons are to emerge, how scattering matrices are to be computed, how Feynman diagrams are to dictate dynamics and virtual processes are to give radiative corrections. To use a well-known phrase ${ }^{9}$, the emphasis on geometry in the canonical program 'drove a wedge between general relativity and the theory of elementary particles'.

In the covariant approach ${ }^{4,10,11}$ the emphasis is just the opposite. (Incidentally, in the context of quantum gravity, the term 'covariant' is somewhat misleading because the introduction of a background metric violates diffeomorphism covariance. It is used mainly to emphasize that this approach does not involve a $3+1$ decomposition of space-time.) Field-theoretic techniques are put at the forefront. The first step in this program is to split the space-time metric $g_{\mu \nu}$ in two parts, $g_{\mu \nu}=\eta_{\mu \nu}+\sqrt{G} h_{\mu v}$, where $\eta_{\mu \nu}$ is to be a background, kinematical metric, often chosen to be flat, $G$ is Newton's constant, and $h_{\mu v}$, the deviation of the physical metric from the chosen background, the dynamical field. The two roles of the metric tensor are now split. The overall attitude is that this sacrifice of the fusion of gravity and geometry is a moderate price to pay for ushering-in the powerful machinery of perturbative quantum field theory. Indeed, with this splitting most of the conceptual problems discussed above seem to melt away. Thus, in the transition to the quantum theory it is only $h_{\mu \nu}$ that is quantized. Quanta of this field propagate on the classical background space-time with metric $\eta_{\mu v}$. If the background is in fact chosen to be flat, one can use the Casimir operators of the Poincaré group and show that the quanta have spin two and rest mass zero. These are the gravitons. The Einstein-Hilbert Lagrangian tells us how they interact with one another. Thus, in this program, quantum general relativity was first reduced to a quantum field theory in Minkowski space. One could apply to it all the machinery of perturbation theory that had been so successful in particle physics. One now had a definite program to compute amplitudes for various scattering processes. Unruly gravity appeared to be tamed and forced to fit into the mold created to describe quantum electromagnetic interactions. Thus, the covariant quantization program was more in tune with the mainstream developments in physics at the time. In the early sixties, Gupta and Feynman outlined an extension of perturbative methods from quantum electrodynamics to gravity. A few years later DeWitt carried this analysis to completion by systematically formulating the Feynman rules for calculating scattering amplitudes among gravitons and between gravitons and matter quanta. He showed that the theory is unitary order by order in the perturbative expansion. By the early seventies, the covariant approach had led to several concrete results ${ }^{10}$.

Consequently, the second stage of the covariant program began with great enthusiasm and hope. The motto was: Go forth, perturb, and expand. The enthusiasm was first generated by the discovery that Yang-Mills theory coupled to fermions is renormalizable if the masses of gauge particles are generated by a spontaneous symmetry-breaking mechanism. (In fact DeWitt's quantum gravity work ${ }^{10}$ played a seminal role in the initial stages of the extension of perturbative techniques from Abelian to non-Abelian gauge theories.) This led to a successful theory of electroweak interactions. Particle physics witnessed a renaissance of quantum field theory. The enthusiasm spilled over to gravity. Courageous calculations were performed to estimate radiative corrections. Unfortunately, however, this research soon ran into its first road block. The theory was shown to be non-renormalizable when two loop effects are taken into account for pure gravity and already at one loop for gravity coupled with matter ${ }^{12}$. To appreciate the significance of this result, let us return to the quantum theory of photons and electrons. This theory is perturbatively renormalizable. This means that, although individual terms in the perturbation expansion of a physical amplitude may diverge due to radiative corrections involving closed loops of virtual particles, these infinities are of a specific type; they can be systematically absorbed in the values of free parameters of the theory, the fine structure constant and the electron mass. Thus, by renormalizing these parameters, individual terms in the perturbation series can be systematically rendered finite. In quantum general relativity, such a systematic procedure is not available; infinities that arise due to radiative corrections are genuinely troublesome. Put differently, quantum theory acquires an infinite number of undetermined parameters. Although one can still use it as an effective theory in the low energy regime, regarded as a fundamental theory, it has no predictive power at all!

Buoyed, however, by the success of perturbative methods in electroweak interactions, the community was reluctant to give them up in the gravitational case. In the case of weak interactions, it was known for some time that the observed low energy phenomena could be explained using Fermi's simple four-point interaction. The problem was that this Fermi model led to a non-renormalizable theory. The correct, renormalizable model of Glashow, Weinberg and Salam agrees with Fermi's at low energies but marshals new processes at high energies which improve the ultraviolet behaviour of the theory. It was therefore natural to hope that the situation would be similar in quantum gravity. General relativity, in this analogy, would be similar to Fermi's model. The fact that it is not renormalizable 
was taken to mean that it ignores important processes at high energies which are, however, unimportant at low energies, i.e., at large distances. Thus, the idea was that the correct theory of gravity would differ from general relativity but only at high energies, i.e., near the Planck regime. With this aim, higher derivative terms were added to the Einstein-Hilbert Lagrangian. If the relative coupling constants are chosen judiciously, the resulting theory does in fact have a better ultraviolet behaviour. Stelle, Tomboulis and others showed that the theory is not only renormalizable but asymptotically free; it resembles the free theory in the high energy limit. Thus, the initial hope of 'curing' quantum general relativity was in fact realized. However, it turned out that the Hamiltonian of this theory is unbounded from below, and consequently the theory is drastically unstable! In particular, it violates unitarity; probability fails to be conserved. The success of the electroweak theory suggested a second line of attack. In the approaches discussed above, gravity was considered in isolation. The successful unification of electromagnetic and weak interactions suggested the possibility that a consistent theory would result only when gravity is coupled with suitably chosen matter. The most striking implementation of this viewpoint occurred in supergravity. Here, the hope was that the bosonic infinities of the gravitational field would be cancelled by those of suitably chosen fermionic sources, giving us a renormalizable quantum theory of gravity. Much effort went into the analysis of the possibility that the most sophisticated of these theories $-N=8$ supergravity - can be employed as a genuine grand unified theory. (For a number of years, there was a great deal of confidence, especially among particle physicists, that supergravity was on the threshold of providing the complete quantum gravity theory. For instance, in the centennial celebration of Einstein's birthday at the Institute of Advanced Study, Princeton ${ }^{13}$ - the proceedings of which were videotaped and archived for future historians and physicists there were two talks on quantum gravity, both devoted to supergravity. A year later, in his Lucasian Chair inaugural address Hawking ${ }^{14}$ suggested that end of theoretical physics was in sight because $N=8$ supergravity was likely to be the final theory.) It turned out that some cancellation of infinities does occur and that supergravity is indeed renormalizable to two loops even though it contains matter fields coupled to gravity. Furthermore, its Hamiltonian is manifestly positive and the theory is unitary. However, it is believed that at fifth and higher loops it is again non-renormalizable.

\section{Paradigm shifts}

By and large, the canonical approach was pursued by relativists and the covariant approach by particle physicists. In the mid-eighties, both approaches received unexpected boosts. These launched the third phase in the development of quantum gravity.
A group of particle physicists had been studying string theory to analyse strong interactions from a novel angle. The idea was to replace point particles by 1-dimensional extended objects - strings - and associate particle-like states with various modes of excitations of the string. Initially there was an embarrassment: in addition to the spin-1 modes characteristic of gauge theories, string theory included also a spin-2, massless excitation. But it was soon realized that this was a blessing in disguise: the theory automatically incorporated a graviton. In this sense, gravity was already built into the theory! However, it was known that the theory had a potential quantum anomaly which threatened to make it inconsistent. In the mid-eighties, Green and Schwarz showed that there is an anomaly cancellation. Perturbative string theory could be consistent in certain space-time dimensions - 26 for a purely bosonic string and 10 for a superstring ${ }^{15,16}$. Since strings were assumed to live in the background of Minkowski space-time, one could apply perturbative techniques. However, in this reincarnation, the covariant approach underwent a dramatic revision. Since it is a theory of extended objects rather than point particles, the quantum theory has brand new elements; it is no longer a local quantum field theory. The field theoretic Feynman diagrams are replaced by world-sheet diagrams. This replacement dramatically improves the ultraviolet behaviour and, although explicit calculations have been carried out only at 2 or 3 loop order, it is widely believed that the perturbation theory is finite to all orders; it does not even have to be renormalized. The theory is also unitary. It has a single, new fundamental constant - the string tension - and, since various excited modes of the string represent different particles, there is a built-in principle for unification of all interactions! Although none of the known low energy reductions appears to correspond to the world we actually observe, string theory has provided us with a glimpse of an entirely new vista: the concrete possibility that unification could be brought about by a tightly woven, non-local theory. From the viewpoint of local quantum field theories that particle physicists have used in studying electroweak and strong interactions, this mathematical structure seems almost magical. Therefore there is a hope in the string community that this theory would encompass all of fundamental physics; it would be the 'theory of everything'.

Unfortunately, it soon became clear that string perturbation theory also faces some serious limitations. Perturbative finiteness would imply that each term in the perturbation series is ultra-violet finite. (Incidentally infrared divergences appear to persist. While this is an important limitation from the mathematical physics perspective, as in QED, these are regarded as 'harmless' for calculation of physical effects. I thank Ashoke Sen for discussions on this issue.) However Gross and Periwal have shown that in the case of bosonic strings, when summed, the series diverges and does so uncontrollably. (Technically, it is not even 'Borel-summable'.) They also gave arguments 
that the conclusion would not be changed if one uses superstrings instead. Independent support for these arguments has come from work on random surfaces due to Ambjorn and others. One might wonder why the divergence of the sum should be regarded as a serious failure of the theory. After all, in quantum electrodynamics, the series is also believed to diverge. Recall, however, that quantum electrodynamics is an inherently incomplete theory. It ignores many processes that come into play at high energies or short distances. In particular, it completely ignores the microstructure of space-time and simply assumes that spacetime can be approximated by a smooth continuum even below the Planck scale. Therefore, it can plead incompleteness and shift the burden of this infinity to a more complete theory. A 'theory of everything' on the other hand, has nowhere to hide. It cannot plead incompleteness and shift its burden. It must face the Planck regime squarely. So, if string theory is to be consistent, it must have key non-perturbative structures. The current and the fourth stage of the particle physics motivated approaches to quantum gravity is largely devoted to unravelling such structures and using them to address some of the outstanding physical problems.

On the relativity side, the third stage also began with an unexpected but innocuous-sounding observation: the geometrodynamics program laid out by Dirac, Bergmann, Wheeler and others simplifies significantly if we regard a connection - rather than the 3-metric - as the basic object ${ }^{17}$. While metrics determine distances and angles, connections enable one to 'parallel transport' objects along curves. A familiar example from text-book quantum mechanics is the electro-magnetic vector potential $A$ that lets us transport the wave function $\psi(x)$ of a charged particle, such as the electron, from one point to another along any given curve: under an infinitesimal displacement, while the change in the wave function of an uncharged particle is given just by $\Delta \vec{x} \cdot \vec{\nabla} \psi(x)$, for a charged particle, it is given by $\Delta \vec{x} \cdot(\vec{\nabla}-(i q / \hbar) \vec{A}) \psi(x)$ where $q$ is the charge of the particle. The presence of a non-zero $\vec{A}$ manifests itself in a change of phase of $\psi$, the most dramatic example of which occurs in the celebrated Bohm-Aharanov effect. In QCD the (matrix-valued) vector potentials couple similarly to the wave functions of quarks and dictate the change of their state as one moves from one point to another. In the gravitational context, the most familiar connection is the one introduced by Levi-Civita which enables one to parallel transport a vector on a curved manifold. We now know that, in their quest for an unified field theory, Einstein and Schrödinger, among others, had recast general relativity as a theory of Levi-Civita connections (rather than metrics) already in the fifties ${ }^{18}$. However, the theory became rather complicated.

This episode had been forgotten and connections were re-introduced in the mid-eighties. However, now these were 'spin-connections', required to parallel propagate spinors, such as the left handed fermions used in the stan- dard model of particle physics ${ }^{17,19}$. Rather than making the theory complicated, these connections simplify Einstein's equations considerably. For example, the dynamics of general relativity can now be visualized simply as a geodesic motion on the space of spin-connections (with respect to a natural metric extracted from the constraint equations). Since general relativity is now regarded as a dynamical theory of connections, this reincarnation of the canonical approach is called connection-dynamics.

Perhaps the most important advantage of the passage from metrics to connections is that the phase-space of general relativity is now the same as that of gauge theories ${ }^{17,19}$. The 'wedge between general relativity and the theory of elementary particles' that Weinberg referred to is largely removed without sacrificing the geometrical essence of general relativity. One could now import into general relativity techniques that have been highly successful in the quantization of gauge theories. At the kinematic level, then, there is a unified framework to describe all four fundamental interactions. The dynamics, of course, depends on the interaction. In particular, while there is a background space-time geometry in electroweak and strong interactions, there is none in general relativity. Therefore, qualitatively new features arise. These were exploited in the late eighties and early nineties to solve simpler models - general relativity in $2+1$ dimensions ${ }^{17,20}$; linearized gravity clothed as a gauge theory ${ }^{17}$; and certain cosmological models. To explore the physical, $3+1$ dimensional theory, a 'loop representation' was introduced by Rovelli and Smolin ${ }^{21}$. Here, quantum states are taken to be suitable functions of loops on the 3-manifold. (This is the origin of the name 'loop quantum gravity'. The loop representation played an important role in the initial stages. Although this is no longer the case in the current, fourth phase, the name is still used to distinguish this approach from others.) This led to a number of interesting and intriguing results, particularly by Gambini, Pullin and their collaborators, relating knot theory and quantum gravity ${ }^{22}$. Thus, there was rapid and unanticipated progress in a number of directions which rejuvenated the canonical quantization program. Since the canonical approach does not require the introduction of a background geometry or use of perturbation theory, and because one now has access to fresh, non-perturbative techniques from gauge theories, in relativity circles there is a hope that this approach may lead to well-defined, non-perturbative quantum general relativity (or its supersymmetric version, supergravity).

However, a number of these considerations remained rather formal until the mid-nineties. Passage to the loop representation required an integration over the infinite dimensional space of connections and the formal methods were insensitive to possible infinities lurking in the procedure. Indeed, such integrals are notoriously difficult to perform in interacting field theories. To pay due respect to the general covariance of Einstein's theory, one needed diffeomorphism invariant measures and there were 
folk-theorems to the effect that such measures did not exist!

Fortunately, the folk-theorems turned out to be incorrect. To construct a well-defined theory capable of handling field theoretic issues, a quantum theory of Riemannian geometry was systematically constructed in the midnineties ${ }^{23}$. This launched the fourth (and the current) stage in the canonical approach. Just as differential geometry provides the basic mathematical framework to formulate modern gravitational theories in the classical domain, quantum geometry provides the necessary concepts and techniques in the quantum domain. Specifically, it enables one to perform integration on the space of connections for constructing Hilbert spaces of states and to define geometric operators corresponding, e.g. to areas of surfaces and volumes of regions (even though the classical expressions of these quantities involve non-polynomial functions of the Riemannian metric). There are no infinities. One finds that, at the Planck scale, geometry has a definite discrete structure. Its fundamental excitations are 1-dimensional, rather like polymers, and the space-time continuum arises only as a coarse-grained approximation. The fact that the structure of space-time at Planck scale is qualitatively different from Minkowski background used in perturbative treatments reinforced the idea that quantum general relativity (or supergravity) may well be non-perturbatively finite.

Finally, quantum geometry is a general framework that is not tied down to general relativity (or supergravity). However, since general relativity is the best classical theory of gravity we have, it is well worth investigating, at least as the first step, whether quantum general relativity exists non-perturbatively. Much of research in loop quantum gravity has been focussed on this question. Quantum geometry effects have already been shown to resolve the bigbang singularity and solve some of the long-standing problems associated with black holes.

\section{The past decade}

The first three stages of developments in quantum gravity taught us many valuable lessons. Perhaps the most important among them is the realization that perturbative, field theoretic methods which have been so successful in other branches of physics are not as useful in quantum gravity. The assumption that space-time can be replaced by a smooth continuum at arbitrarily small scales leads to inconsistencies. We can neither ignore the microstructure of space-time nor presuppose its nature. We must let quantum gravity itself reveal this structure to us. Irrespective of whether one works with strings or supergravity or general relativity, one has to face the problem of quantization non-perturbatively. In the current, fourth stage both approaches have undergone a metamorphosis. The covariant approach has led to string theory and the canonical approach developed into loop quantum gravity. The mood seems to be markedly different. In both approaches, non-perturbative aspects are at the forefront and conceptual issues are again near center-stage. However, there are also key differences. Most work in string theory involves background fields and uses higher dimensions and supersymmetry as essential ingredients. The emphasis is on unification of gravity with other forces of Nature. Loop quantum gravity, on the other hand, is manifestly background independent. Supersymmetry and higher dimensions do not appear to be essential. However, it has not provided any principle for unifying interactions. In this sense, the two approaches are complementary rather than in competition. Each provides fresh ideas to address some of the key problems but neither is complete.

In the rest of this section, I will illustrate the current developments by sketching a few of the more recent results. In the case of string theory, my discussion will be very brief because these topics are discussed in greater detail by several other articles in this issue.

\section{String theory}

Over the past decade, novel non-perturbative ideas have been introduced in string theory. Unlike in the perturbative epoch, it is no longer a theory only of one dimensional extended objects. Higher dimensional objects, called 'branes' have played an increasingly important role. Although for historical reasons it is still called 'string theory', from a fundamental, conceptual perspective, strings are no more basic than branes. Of particular interest are the D-branes introduced by Polchinski on which open strings satisfying 'Dirichlet type' boundary conditions can end (whence the adjective ' $D$ '). These lie at the heart of the statistical mechanical calculation of entropy of large extremal black holes in string theory.

The second key development was even more radical: Maldecena made the bold proposal that string theory on a certain anti-De sitter background space-time is isomorphic with a gauge theory living on its boundary. In the first and the most studied version, the background space-time is assumed to be a product of a five dimensional anti-De Sitter space-time with a five-dimensional sphere (whose radius equals the cosmological radius of the anti-De Sitter space-time) while the gauge theory lives on the 4-dimensional boundary of the five-dimensional anti-De Sitter space-time. Since then the setup has been generalized to various non-compact dimensions. The boundary conditions - and hence the resulting string theories - are not of direct physical interest because our universe has a positive, rather than a negative, cosmological constant and because the compact spheres do not represent microscopic 'curled-up' dimensions because they now have huge radii. Nonetheless, from a mathematical physics perspective, the proposed duality is fascinating because it relates a 
'gravity theory' residing on a curved space-time with a qualitatively different 'gauge theory' living on a (conformally) flat space-time. It has had some powerful applications, e.g., in unravelling the structure of certain supersymmetric gauge theories through supergravity!

Finally there are interesting proposals relating various types of string theories that go under the name dualities. Although there are no conclusive proofs, these ideas suggest that the five perturbatively constructed string theories and supergravity may be special limits of a grander, unknown theory, generally referred to as the $M$ theory. The scenario has generated a great deal of enthusiasm. For, the conjectured theory is likely to be very rich. In particular, it should provide isomorphisms between the strong coupling regime of one string theory to the weak coupling regime of another. Unravelling of its non-perturbative structures will undoubtedly provide qualitatively new insights and perhaps even radically change our current perspectives.

\section{Loop quantum gravity}

Over the past decade, the main thrust of research in loop quantum gravity has been on using quantum geometry to address some of the long standing problems in the field. Certain key techniques introduced by Thiemann have provided glimpses of the qualitative changes in quantum dynamics that occur because of the absence of a background geometry. Specifically, thanks to the fundamental discreteness of quantum Riemannian geometry, the ultraviolet divergences - also in the definition of matter Hamiltonians are naturally tamed ${ }^{23,24}$. However, in the full theory, two major issues still remain. First, there is a large number of ambiguities in the formulation of quantum Einstein's equations and one needs additional inputs to remove them. Second, it is still not clear whether any of the current formulations admits a semi-classical sector that reproduces the low energy world around us. (Several different avenues are being pursued to address these issues ${ }^{23}$. These include the 'discrete approach' due to Gambini and Pullin ${ }^{25}$ in which one discretizes the theory prior to quantization; spin-foam approaches due to Baez, Barrett, Crane, Perez, Rovelli and others ${ }^{26,27}$ in which one uses a background independent, the path integral analog of loop quantum gravity; and the 'master constraint program' of Dittrich and Thiemann ${ }^{28}$ which uses some of the key ideas of Klauder' ${ }^{29}$ affine quantum gravity program.) So far, advances of direct physical interest have occurred by adopting a strategy which has been effective also in string theory: isolate and analyse issues on which significant progress can be made in spite of the gaps in the understanding of the full theory. In the rest of this sub-section, I will illustrate how this strategy is implemented. Rather than describing several results briefly, I will focus just on one. This will enable me to provide some details that are necessary for the reader to appreciate the subtle manner in which quantum geometry effects operate.
The issue in question is the nature of the quantum big bang. Most work in cosmology is carried out in the context of spatially homogeneous and isotropic models and perturbations thereof ${ }^{30}$. In the simplest model, the basic variables of the symmetry reduced classical system are the scale factor $a$ and matter fields $\phi$. Symmetries imply that space-time curvature goes as $\sim 1 / a^{n}$, where $n>0$ depends on the matter field under consideration. Einstein's equations then predict a big-bang, where the scale factor goes to zero and the curvature blows up. Space-time comes to an end and the classical physics stops. For over three decades a key question has been: Can these 'limitations' of general relativity be overcome in an appropriate quantum theory? In traditional quantum cosmologies, the answer is in the negative. Typically, to resolve the singularity one either has to use matter (or external clocks) with unphysical properties or introduce additional boundary conditions, e.g., by invoking new principles, that dictate how the universe began.

In a series of papers Bojowald, Ashtekar, Date, Hossain, Lewandowski, Maartens, Singh, Vandersloot and others have shown that the situation in loop quantum cosmology is quite different: the underlying quantum geometry makes a qualitative difference very near the big-bang ${ }^{23,31}$. At first, this seems puzzling because after symmetry reduction, the system has only a finite number of degrees of freedom. Thus, quantum cosmology is analogous to quantum mechanics rather than quantum field theory. How then can one obtain qualitatively new predictions? The answer is quite surprising: if one follows the program laid out in the full theory, then even for the symmetry reduced model one is led to a new quantum mechanics! Specifically, the representation (of the observable algebra) that naturally arises in loop quantum cosmology is inequivalent to that used in the older, traditional quantum cosmology. And in the new representation, quantum evolution is well-defined right through the big-bang singularity.

More precisely, the situation in dynamics can be summarized as follows. Because of the underlying symmetries, dynamics is dictated just by one of the ten Einstein equations, called the Hamiltonian constraint. Let us consider the simplest case of homogeneous, isotropic cosmologies coupled to a scalar field. In traditional quantum cosmology, this constraint is the celebrated Wheeler-DeWitt equa$\operatorname{tion}^{7,8}$ - a second order differential equation on wave functions $\psi(a, \phi)$ that depend on the scale factor $a$ and the scalar field $\phi$. Unfortunately, some of the coefficients of these equations diverge at $a=0$, making it impossible to obtain an unambiguous evolution across the singularity. In loop quantum cosmology, the scale factor naturally gets replaced by $\mu$, the momentum conjugate to the connection. $\mu$ ranges over the entire real line and is related to the scale factor via $|\mu|=$ const $a^{2}$. Negative values of $\mu$ correspond to the assignment of one type of spatial orientation, positive to the opposite orientation, and $\mu=0$ corresponds to the degenerate situation at the singularity. The Wheeler- 
DeWit equation is now represented by a difference equation on the quantum state $\psi(\mu, \phi)$ :

$$
\begin{aligned}
& C^{+}(\mu) \psi\left(\mu+4 \mu_{0}, \phi\right)+C^{0}(\mu) \psi(\mu, \phi) \\
& +C^{-}(\mu) \psi\left(\mu-4 \mu_{0}, \phi\right)=\ell_{\mathrm{Pl}}^{2} \hat{H}_{\phi} \psi(\mu, \phi)
\end{aligned}
$$

where $C^{ \pm}(\mu), C^{0}(\mu)$ are fixed functions of $\mu ; \mu_{0}$, a constant, determined by the lowest eigenvalue of the area operator and $\hat{H}_{\phi}$ is the matter Hamiltonian. Again, using the ana$\log$ of the Thiemann regularization from the full theory, one can show that the matter Hamiltonian is a well-defined operator.

Primarily, eq. (1) is the quantum Einstein's equation that selects the physically permissible $\psi(\mu, \phi)$. However, if we choose to interpret $\mu$ as a heuristic time variable, eq. (1) can be interpreted as an 'evolution equation' which evolves the state through discrete time steps. The highly non-trivial result is that the coefficients $C^{ \pm}(\mu), C^{0}(\mu)$ are such that one can evolve right through the classical singularity, i.e., right through $\mu=0$. Since all solutions have this property, the classical singularity is resolved. However, to complete the quantization program, one has to introduce the appropriate scalar product on the space of solutions to the constraint, define physically interesting operators on the resulting Hilbert space $\mathrm{H}_{\text {final }}$ and examine their expectation values and fluctuations, especially near the singularity.

All these steps have been carried out in detail in the case when $\phi$ is a massless scalar field ${ }^{32}$ (The extension of the analysis to include potential terms for the matter field $\phi$ or anisotropies for the combined system involves only technical complications. The overall conceptual picture remains the same.). Specifically, in each classical solution, $\phi$ is a monotonic function of time. Therefore, one can regard it as an 'internal clock' with respect to which the scale factor evolves. With this interpretation, the discrete eq. (1) takes the form $\partial_{t}^{2} \psi=-\Theta \psi$, where $\Theta$ is a selfadjoint (difference) operator, independent of $\phi \sim t$. This is precisely the form of the Klein-Gordon equation in static space-times. (In technical terms, this provides a satisfactory 'deparametrization' of the theory.) Therefore, one can use techniques from quantum field theory in static spacetimes to construct an appropriate inner product and define a complete family of ('Dirac') observables. Using the two, one can construct semi-classical states - analogs of coherent states of a harmonic oscillator - and write down explicit expressions for expectation values and fluctuations of physical observables in them. As one might expect, the evolution is well-defined across the singularity but quantum fluctuations are huge in its neighbourhood.

Now that there is a well-defined theory, one can use numerical methods to evolve quantum states and compare quantum dynamics with the classical one in detail. Since we do not want to make a priori assumptions about what the quantum state was at the big-bang, it is best to start the evolution not from the big bang but from late times ('now'). Consider then wave functions which are sharply peaked at a classical trajectory at late times and evolve them backward. The first question is: how long does the state remain semi-classical? A pleasant surprise is that it does so till very early times - essentially till the epoch when the matter density reaches the Planck density. Now, this is precisely what one would physically expect. However, with a complicated difference equation such as (1), a priori there is no guarantee that semi-classicality would not be lost very quickly. In particular, this result provides support for the standard practice, e.g., in inflationary models, of assuming a classical continuum in the very early universe. Next, one can ask what happens to the quantum state very near and beyond the big-bang. As explained above, the state loses semi-classicality (i.e. fluctuations become large) near the big-bang. Does it then remain in a 'purely quantum regime' forever or does it again become semi-classical beyond a Planck regime on the 'other side' of the big bang? This is a question that lies entirely outside the domain of the standard Wheeler-Dewitt equation because it loses predictivity at the big-bang. In loop quantum cosmology, on the other hand, the evolution is well-defined and completely deterministic also beyond the big-bang. A priori there is no way to know what the answer would be. Space-time may well have been a 'quantum foam' till the big-bang and classicality may then have emerged only after the big-bang. Or, there may have been a classical space-time also on the 'other side'. Detailed numerical calculations show that the wave function becomes semi-classical again on the other side; gravity becomes repulsive in the Planck regime, giving rise to a 'bounce'. Thus, loop quantum cosmology predicts that the universe did not originate at the big bang but has a long prior history. Through quantum dynamics, the universe tunnels from a contracting phase in the distant past ('before the bang') to an expanding phase in the distant future ('now') in a specific manner. Classically, of course such a transition is impossible.

To summarize, the infinities predicted by the classical theory at the big-bang are artifacts of assuming that the classical, continuum space-time approximation is valid right up to the big-bang. In the quantum theory, the state can be evolved through the big-bang without any difficulty. However, the classical, continuum completely fails near the big-bang; figuratively, the classical space-time 'dissolves'. This resolution of the singularity without any 'external' input (such as matter violating energy conditions) is dramatically different from what happens with the standard Wheeler-DeWitt equation of quantum geometrodynamics $^{1,3,4,7-10}$. However, for large values of the scale factor, the two evolutions are close; as one would have hoped, quantum geometry effects intervene only in the 'deep Planck regime' resulting in a quantum bridge connecting two classically disconnected space-times. From this perspective, then, one is led to say that the most striking of the 


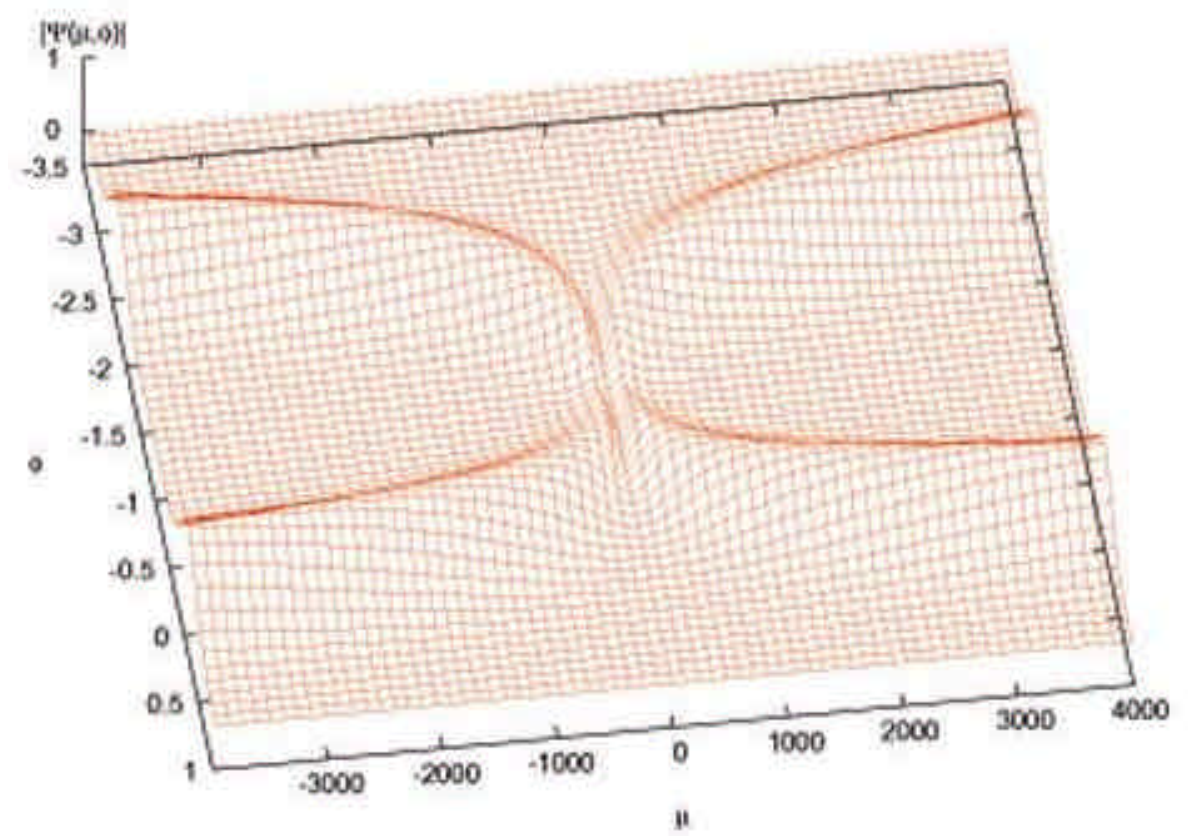

Figure 1. Comparison between quantum and classical evolutions via plot of $|\psi(\mu, \phi)|$. Since $\mu \rightarrow-\mu$ changes only the spatial orientation, it suffices to consider just $\mu \geq 0$. Except in the Planck regime very near $\mu=0$, $\psi$ is sharply peaked at the classical trajectories. But the trajectory in the top half represents an expanding universe while that in the bottom half, a contracting universe. Thus, quantum geometry in the Planck regime bridges two vast but classically disjoint space-times.

consequences of loop quantum gravity are not seen in older approaches because they 'wash out' the fundamental discreteness of quantum geometry.

\section{Outlook}

The road to quantum gravity has been long, spanning some four decades. Along the way came many new insights, jubilations as well as frustrations. Because of the page limit, I could only provide a general flavour of these trials, tribulations and triumphs. In particular, I had to restrict myself to the 'main-stream' programs whose development can be continuously tracked over several decades. There also exist a number of other fascinating and highly original approaches - particularly causal dynamical triangulations $^{33,34}$, Euclidean quantum gravity ${ }^{35,36}$, twistor theory ${ }^{37,38}$ and the related theory of $\mathrm{H}$-spaces ${ }^{39}$, asymptotic quantization $^{40}$, non-commutative geometry ${ }^{41}$ and causal sets ${ }^{42}-$ that I could not discuss. (Accounts of the present status of several of these approaches can be found in the articles by Dowker, Ford, Gambini and Pullin and Penrose in ref. 43.)

But I hope I managed to convey that, in spite of all the twists and turns in the winding road, there have been definite advances. We have learned that, because relativistic gravity is so deeply intertwined with space-time geometry, quantum gravity has unforeseen dimensions that would have surprised even the great leaders of the early period. We tried hard to extend perturbative methods of local quantum field theory which have been so successful in
QED $^{1}$. The efforts did lead to a successful perturbative framework for non-Abelian gauge theories ${ }^{10,12}$. But we found conclusive evidence that these methods are insufficient for quantum gravity: they lead to uncontrollable ultraviolet divergences ${ }^{12}$. Thanks to string theory, we now have, for the first time, a concrete alternative - a computational framework to calculate scattering amplitudes which yields finite results to any order in perturbation theory ${ }^{16}$. Furthermore, the theory provides a brand new avenue to the unification of all interactions; the plethora of elementary particles is now reduced to various vibrational modes of the superstring. For decades we have been troubled by the fact that space-time of general relativity comes to an abrupt end at singularities and classical physics literally stops there. Loop quantum gravity has shown that this is an artifact of pushing the classical theory beyond the domain of its validity ${ }^{23,31}$. Quantum geometry extends its life. What we thought of as a 'tiny, Planck scale region' can actually be a bridge joining our space-time to another vast classical region $^{32}$; quantum space-time may be vastly larger than what general relativity had us believe. (In section IV B I discussed cosmological singularities. However, the situation is similar also for space-like black hole singularities ${ }^{44,45}$ ) Finally, both approaches have provided fascinating insights into the nature of quantum black holes ${ }^{23,46}$, a topic that would require a separate article in its own right.

All currently active directions point to the necessity of radical revisions of the 20th century paradigm of theoretical physics. String theory abandons local quantum field theories altogether and focuses instead on the study 
of extended quantum objects. Loop quantum gravity asks us to forego our cherished space-time continuum and embrace a quantum geometry instead. Twistor theory and non-commutative geometry suggest that we abandon the familiar space-time already at the classical level and reformulate general relativity well before the word 'quantum' is uttered or the symbol $\hbar$ introduced. No matter which of these approaches find an expression in the final quantum gravity theory, it is clear that quantum gravity will have deep ramifications on all of fundamental physics. General relativity led to a radical revision of our notions of space and time, thereby reshaping the conceptual foundation of all of physics. Impact of the successful quantum gravity theory on fundamental physics will be even deeper.

But today we cannot be certain which - or indeed, any of these directions will constitute major components of the final theory. Thanks to the sustained work spanning many decades, most notable advances have occurred in the 'covariant' and 'canonical' approaches. However, even here one encounters serious incompleteness and some troubling features. How is the supersymmery broken in string theory and how does reduction to four large dimensions occur? While the theory is very tight in terms of its fundamental constants, there is a huge freedom in the choice of 'moduli-parameters'. There appear to be over $10^{200}$ 'vacua', each giving rise to its own low energy theory! How is this freedom to be reduced? No compelling principle seems to be in sight. More generally, our understanding of the presumed M-theory is very incomplete. Incompleteness also pervades loop quantum gravity. How are the ambiguities in the formulation of quantum Einstein's equations removed in the full theory? Does this theory admit a viable semi-classical sector? Through minisuperspaces we have learned that cosmological and black hole singularities are resolved through quantum geometry effects and loop quantum gravity enables one to perform a deterministic evolution across these singularities. Do these features survive beyond the minisuperspace approximation? How do inhomogeneous perturbations evolve in the cosmological context? Is this evolution compatible with observational constraints on structure formation? While there is vigorous ongoing research to answer such questions in both approaches, one cannot say that a satisfactory resolution is imminent. Even more important is the issue of observations. So far, not a single non-trivial and firm prediction of any quantum gravity theory has been verified directly. Therefore, as we celebrate the 100th anniversary of Einstein's Annus Mirabilis it is important that we maintain a long range perspective and not repeat our past error of overconfidence. In particular, we would do well to avoid the traps that the celebrated biologist François $\mathrm{Jacob}^{47}$ warned all scientists about:

'The danger for scientists is not to measure the limits of their science, and thus their knowledge. This leads to mix what they believe and what they know. Above all, it creates the certitude of being right [prematurely].'

1. Isham, C. J., An introduction to quantum gravity. In Quantum Gravity, An Oxford Symposium (eds Isham, C. J., Penrose, R. and Sciama, D. W.), Clarendon Press, Oxford, 1975.

2. Arnowitt, R., Deser, S. and Misner, C. W., The dynamics of general relativity. Gravitation: An Introduction to Current Research (ed. Witten, L.), John Wiley, New York, 1962.

3. Komar, A., Quantization program for general relativity. Relativity (eds Carmeli, M., Fickler, S. I. and Witten, L.), Plenum, New York, 1970.

4. Ashtekar, A. and Geroch, R., Quantum theory of gravitation. Rep. Prog. Phys., 1974, 37, 1211-1256.

5. Bergmann, P. G. and Komar, A., The phase space formulation of general relativity and approaches toward its canonical quantization. General Relativity and Gravitation, On Hundred Years after the Birth of Albert Einstein (ed. Held, A.), Plenum, New York, 1980.

6. Kuchaø, K., Canonical methods of quantization. Quantum Gravity 2, A Second Oxford Symposium (eds Isham, C. J., Penrose, R. and Sciama, D. W.), Clarendon Press, Oxford, 1981.

7. Wheeler, J. A., Geometrodynamics, Academic Press, New York, 1962.

8. Wheeler, J. A., Geometrodynamics and the issue of the final state. Relativity, Groups and Topology (eds DeWitt, C. M. and DeWitt, B. S.), Gordon and Breach, New York, 1964.

9. Weinberg, S., Gravitation and Cosmology, John Wiley, New York, 1972.

10. DeWitt, B. S., Covariant quantum geometrodynamics. In Magic Without Magic: John Archibald Wheeler (ed. Klauder, J. R.), W. H. Freeman, San Fransisco, 1972.

11. Duff, M., Covariant qauantization. Quantum Gravity, An Oxford Symposium (eds Isham, C. J., Penrose, R. and Sciama, D. W.), Clarendon Press, Oxford, 1975.

12. Isham, C. J., Quantum gravity - An overview. Quantum Gravity 2, A Second Oxford Symposium (eds Isham, C. J., Penrose, R. and Sciama, D. W.), Clarendon Press, Oxford, 1981.

13. Wolf, H. (ed.), Some Strangeness in Proportion, Addison Wesley, Reading, 1980.

14. Hawking, S. W., Is End In Sight for Theoretical Physics?: An Inaugural Address, Cambridge UP, Cambridge, 1980.

15. Greene, M. B., Schwarz, J. H. and Witten, E., Superstring Theory, Cambridge UP, Cambridge, 1987, vols 1, 2.

16. Polchinski, J., String Theory, Cambridge UP, Cambridge, 1998, vols $1,2$.

17. Ashtekar, A., Lectures on Non-perturbative Canonical Gravity, Notes prepared in collaboration with R. S. Tate, World Scientific, Singapore, 1991.

18. Ashtekar, A., Quantum mechanics of geometry. The Universe: Visions and Perspectives (eds Dadhich, N. and Kembhavi, A.), Kluwer Academic, Dordrecht, gr-qc/9901023, 2000.

19. Baez, J. and Muniain, J. P., Gauge Fields, Knots and Gravity, World Scientific, Singapore, 1994.

20. Ashtekar, A., Mathematical problems of non-perturbative quantum general relativity. Gravitation and Quantizations. Proceedings of the 1992 Les Houches summer school (eds Julia, B. and ZinnJustin, J.), Elsevier, Amsterdam; also available as gr-qc/9302024.

21. Rovelli, C., Loop quantum gravity. Living Rev. Rel., 1998, 1, 1.

22. Gambini, R. and Pullin, J., Loops, Knots, Gauge Theories and Quantum Gravity, Cambridge UP, Cambridge, 1996.

23. Ashtekar, A. and Lewandowski, L., Background independent quantum gravity: A status report. Class. Quant. Grav., 2004, 21, R53-R152. 
24. Thiemann, T., Introduction to Modern Canonical Quantum General Relativity, Cambridge University Press, Cambridge; draft available as gr-qc/0110034, 2005.

25. Gambini, R. and Pullin, J., Consistent discretizations and quantum gravity gr-qc/0408025, 2004.

26. Perez, A., Spin foam models for quantum gravity. Class. Quant. Grav., 2003, 20, R43-R104.

27. Rovelli, C., Quantum Gravity, Cambridge University Press, Cambridge, 2004.

28. Thiemann, T., The phoenix project: Master constraint programme for loop quantum gravity, gr-qc/0305080, 2003.

29. Klauder, J., Affine quantum gravity. Int. J. Mod. Phys., 2003, D12, 1769-1774.

30. Liddle, A. R. and Lyth, D. H., Cosmological Inflation and Large Scale Structure, Cambridge UP, Cambridge, 2000.

31. Bojowald, M. and Morales-Tecotl, H. A., Cosmological applications of loop quantum gravity. Lect. Notes Phys., 2004, 646, 421462, also available at gr-qc/0306008

32. Ashtekar, A., Pawlowski, T. and Singh, P., Quantum nature of the big bang: An analytical and numerical study, IGPG pre-print, 2005.

33. Loll, R., Discrete approaches to quantum gravity in four dimensions. Living Rev. Rel., 1998, 1, 13.

34. Ambjorn, J., Jurkiewicz, J. and Loll, R., Emergence of a 4D world from causal quantum gravity, hep-th/0404156, 2004.

35. Hawking, S. W. and Israel, W. (eds), General Relativity, An Einstein Centenary Survey, Cambridge UP, Cambridge, 1980.

36. Perini, D., The Asymptotic Safety Scenario For Gravity and Matter, $\mathrm{Ph}$ D dissertation, SISSA, 2004.

37. Penrose, R., Twistor theory, its aims and achievements. Quantum Gravity, An Oxford Symposium (eds Isham, C. J., Penrose, R. and Sciama, D. W.), Clarendon Press, Oxford, 1975.

38. Penrose, R. and Rindler, W., Spinors and Space-times, Cambridge University Press, Cambridge, 1988, vol. 2.
39. Ko, M., Ludvigsen, M., Newman, E. T. and Tod, P., The theory of H space. Phys. Rep., 1981, 71 51-139.

40. Ashtekar, A., Asymptotic quantization, Bibliopolis, Naples, 1984, also available at http://cgpg.gravity.psu.edu/research/asymquantbook.pdf

41. Connes, A., Non-commutative Geometry, Academic Press, New York, 1994.

42. Sorkin, R., Causal sets: discrete gravity, gr-qc/0309009, 2003.

43. Ashtekar, A., 100 Years of Relativity; Space-time Structure: Einstein and Beyond, World Scientific, Singapore, 2005, in press.

44. Ashtekar, A. and Bojowald, M., Quantum geometry and the Schwarzschild singularity, Preprint, 2004.

45. Ashtekar, A. and Bojowald, M., Black hole evaporation: A paradigm. Class. Quant. Grav., 2005, 22, 3349-3362.

46. Horowitz, G. T., Quantum states of black holes. Black Holes and Relativistic Stars (ed. Wald, R. M.), University of Chicago Press, Chicago, 1998.

47. Jacob, F., Of Flies, Men and Mice, Harvard UP, Boston, 1998.

ACKNOWLEDGEMENTS. My understanding of quantum gravity has deepened through discussions with a large number of colleagues. Among them, I especially thank John Baez, Peter Bergmann, Martin Bojowald, Alex Corichi, Klaus Fredenhagen, Rodolfo Gambini, Jim Hartle, Gary Horowitz, Ted Jacobson, Kirill Krasnov, Jerzy Lewandowski, Don Marolf, Jose Mourão, Hermann Nicolai, Roger Penrose, Jorge Pullin, Carlo Rovelli, Hanno Sahlmann, Ashoke Sen, Lee Smolin, Thomas Thiemann, and Madhavan Varadarajan. I would like to thank Naresh Dadhich, Shyam Date and Gary Horowitz for comments that improved the historical account. This work was supported in part by the NSF grant PHY 0090091, the Alexander von Humboldt Foundation, the Eberly research funds of The Pennsylvania State University and the Sir C.V. Raman Chair of the Indian Academy of Sciences. 